\title{
A database of calculated solution parameters for the recently released AlphaFold predicted protein structures
}

Emre Brookes ( $\nabla$ emre.brookes@umontana.edu )

University of Montana

Mattia Rocco

Ospedale Policlinico San Martino

\section{Research Article}

Keywords:

Posted Date: January 12th, 2022

DOl: https://doi.org/10.21203/rs.3.rs-1200245/v1

License: (c) (1) This work is licensed under a Creative Commons Attribution 4.0 International License.

Read Full License

Version of Record: A version of this preprint was published at Scientific Reports on May 5th, 2022. See the published version at https://doi.org/10.1038/s41598-022-10607-z. 
1 A database of calculated solution parameters for the recently released AlphaFold predicted 2 protein structures.

3

4 Emre Brookes ${ }^{1, *}$ and Mattia Rocco ${ }^{2}$

5

$6{ }^{1}$ Department of Chemistry and Biochemistry, The University of Montana, 32 Campus Dr, Missoula,

7 MT 59812, USA. Email: emre.brookes@umontana.edu

$8 \quad{ }^{2}$ Proteomica e Spettrometria di Massa, IRCCS Ospedale Policlinico San Martino, Largo R. Benzi

9 10, I-16132 Genova, Italy (retired, official visitor). Email: mattia.rocco@quipo.it

10

$11{ }^{*}$ Corresponding author 


\section{Abstract}

3 Recent spectacular advances by AI programs in 3D structure predictions from protein sequences

4 have revolutionized the field in terms of accuracy and speed. The resulting "folding frenzy" has

5 already produced predicted protein structure databases for the entire human and other organisms'

6 proteomes. However, rapidly ascertaining a predicted structure's reliability based on measured

7 properties in solution should be considered. Shape-sensitive hydrodynamic parameters such as the

8 diffusion and sedimentation coefficients $\left(D_{t(20, w)}^{0}, s_{(20, w)}^{0}\right)$ and the intrinsic viscosity $([\eta])$ can

9 provide a rapid assessment of the overall structure likeliness, and SAXS would yield the structure-

10 related pair-wise distance distribution function $p(r)$ vs. $r$. Using the extensively validated UltraScan

11 SOlution MOdeler (US-SOMO) suite we have calculated from the AlphaFold structures the

12 corresponding $D_{t(20, w)}^{0}, s_{(20, w)}^{0},[\eta], p(r)$ vs. $r$, and other parameters. Circular dichroism spectra

13 were also computed. The resulting US-SOMO-AF database should aid in rapidly evaluating the

14 consistency in solution of AlphaFold-predicted protein structures. 
1 The Anfinsen dogma, that protein sequences dictates their three-dimensional (3D) structure, was postulated nearly fifty years ago ${ }^{1}$. It set in motion a quest to find methods to reliably and accurately predict 3D protein structures from their sequence, which became even more important with the full sequencing of the human and other genomes (see https://www.ncbi.nlm.nih.gov/genome). Recent spectacular advances in the 3D structure prediction from protein sequences by Artificial Intelligence (AI) programs such as AlphaFold (AF) and RoseTTAfold appear to have revolutionized the field in terms of accuracy and speed ${ }^{2,3}$. Boosted by their success in predicting structures to near (and sometimes even better than) crystallographic accuracy, the AlphaFold consortium (https://alphafold.ebi.ac.uk) has already made publicly available a series of databases of predicted protein structures for the entire human and several other organisms proteomes ${ }^{4}$.

11 However, these AI programs have not tackled the folding issue from a thermodynamic/mechanistic 12 approach, but rather by combining many different observations in a deep learning process 5 . Apart 13 from simple cases of highly homologous sequences, or clearly recognized folding classes, to reasonably rapidly ascertain the degree of confidence of a predicted structure based on a few immediately verify the protein oligomerization state and prompt for the need of further modeling. On a different level, circular dichroism (CD) spectroscopy, possible on very small sample amounts $^{6}$, would permit a rapid check of the actual secondary structure content of a predicted 3D structure.

21 Particularly useful for known single-chain proteins in the AF databases, shape-sensitive hydrodynamic parameters such as the translational diffusion and sedimentation coefficients $\left(D_{t(20, w)}^{0}, s_{(20, w)}^{0}\right)$ and the intrinsic viscosity $([\eta])$, could provide a robust assessment of the overall fold likeliness. These measurements, requiring little material and with a reasonably quick turnaround, are usually accessible in most research endeavors, especially in core facilities where analytical ultracentrifugation ${ }^{7,8}$, multi-angle static and dynamic light scattering (MALS and DLS) coupled to size-exclusion chromatography $(\mathrm{SEC})^{9,10}$ or directly on plate readers ${ }^{11}$, and SEC-coupled 
1 differential viscosimetry ${ }^{12,13}$, can often be found. On another level, small-angle X-ray scattering

2 (SAXS) measurements can provide the rms radius of gyration $R_{g}$ and the pair-wise distance

3 distribution function $p(r)$ vs. $r^{14,15,16}$. Notably, several synchrotron beamlines offer on-line SEC-

4 SAXS (e.g., Table $11.1 \mathrm{in}^{17}$ ), some accepting mailed-in samples for this set-up

5 (e.g., https://www.diamond.ac.uk/Users/Support-for-European-Access-to-Life-Sciences/Applications/Bio-SAXS.html;

6 https://www.embl-hamburg.de/biosaxs/mailin.html).

7 Importantly, all these parameters and functions can be calculated, with varying degree of accuracy,

8 from 3D structures. Among the CD spectra computational methods available, we have chosen

9 SESCA, which appears to offer very accurate results for a wide variety of structures ${ }^{18}$. The

10 computation of the hydrodynamic parameters from atomic level structures is a mature field, with

11 several approaches and corresponding software available, and with an average accuracy comparable

12 to that of the experimentally determined parameters, $2-4 \%{ }^{19,20}$. For the hydrodynamics and the

$13 p(r)$ vs. $r$ distribution calculations, we have employed the extensively validated UltraScan SOlution

14 MOdeler (US-SOMO) public domain suite ${ }^{21,22,23}$.

15 This effort has allowed us to produce and make publicly available, from the AlphaFold released

16 predicted protein structures databases, the comprehensive US-SOMO-AF database presented here,

17 containing the corresponding calculated $M, D_{t(20, w)}^{0}, s_{(20, w)}^{0},[\eta], p(r)$ vs. $r$, CD spectra, and other

18 ancillary information. Note that the AlphaFold databases (https://alphafold.ebi.ac.uk) were

19 generated from the UniProt (https://www.uniprot.org) sequences without any curing. For instance,

20 many proteins are synthesized with a signal peptide ${ }^{24}$, which will be post-translationally removed.

21 This will affect the calculated parameters in an inverse proportion to protein size. As the mature

22 form will be nearly always purified and studied, we have removed the UniProt-identified signal

23 peptide residues from the AlphaFold structures before performing the hydrodynamic, structural and

24 spectroscopic calculations.

25 Based on the calculated values, some analyses regarding the effectiveness of performing a

26 screening of predicted structures against experimental parameters are presented. Advantages,

27 drawbacks, and potential improvements are then discussed. 
Results

Database generation and website implementation

3 The steps leading to the implementation of the US-SOMO-AF database are fully described in the

4 Methods and Online Methods sections. Briefly, each entry in the entire AF database was first compared with the corresponding entry in the UniProt database to find the (putative) signal peptide regions, which were subsequently removed from the AF PDB files. Potential disulfides were

7 identified (allowing a better evaluation of the partial specific volume $\bar{v}$ and of $M$ ) and written as

8 SSBOND records in the cured PDBs, together with HELIX and SHEET information identified

9 using the $\operatorname{DSSP}^{25}$ implementation in UCSF Chimera ${ }^{26}$. Batch-mode US-SOMO was used to

10 calculate $M, \bar{v}, D_{t(20, w)}^{0}, s_{(20, w)}^{0}$, the derived Stokes' radius $R_{s},[\eta], R_{g}$, the maximum extensions

11 along the principal $X, Y$ and $Z$ axes of the molecule, and the generation of the $p(r)$ vs. $r$ distributions

12 (normalized by the $M$ of the structure). SESCA was used to generate 170-270 nm CD spectra.

\begin{tabular}{|c|c|}
\hline \multicolumn{2}{|c|}{ US-SOMO Database } \\
\hline \multicolumn{2}{|c|}{ US-SOMO-AF } \\
\hline \multicolumn{2}{|c|}{$\begin{array}{c}\text { US-SOMO Hydrodynamic, Structural and SESCA CD Calculations on } \\
\text { AlphaFold Predicted Structures }\end{array}$} \\
\hline \multirow[t]{2}{*}{ UniProt accession } & Q9Y5H4-F1 \\
\hline & Search \\
\hline \multirow{2}{*}{$\begin{array}{l}\text { AlphaFold model name } \\
\text { Title }\end{array}$} & AF-Q9Y5H4-F1-model_v1 \\
\hline & $\begin{array}{l}\text { ALPHAFOLD V2.0 PREDICTION FOR } \\
\text { PROTOCADHERIN GAMMA-A1 (Q9Y5H4) }\end{array}$ \\
\hline Source & $\begin{array}{l}\text { MOL_ID: } 1 \\
\text { ORGANISM_SCIENTIFIC: HOMO SAPIENS } \\
\text { ORGANISM_TAXID: } 9606\end{array}$ \\
\hline Signal peptide removed & $1-28$ \\
\hline AlphaFold date & 01-JUL-21 \\
\hline Mean confidence & 75.64 \\
\hline Hydrodynamic, structural and $\mathrm{CD}$ calculations date & 12 -OCT-21 \\
\hline Molecular mass [Da] & 98141.0 \\
\hline Partial specific volume $\overline{\boldsymbol{v}}\left[\mathrm{cm}^{3} / \mathrm{g}\right]$ & 0.732 \\
\hline Translational diffusion coefficient $\boldsymbol{D}^{0}{ }_{20, \mathrm{w}}[\mathrm{F}]$ & 3.26 \\
\hline Sedimentation coefficient $s^{0}{ }_{20, w}[\mathrm{~S}]$ & 3.54 \\
\hline Stokes radius $\boldsymbol{R}_{\boldsymbol{S}}[\mathrm{nm}]$ & 6.56 \\
\hline Intrinsic viscosity [ $\eta\left[\mathrm{cm}^{3} / \mathrm{g}\right]$ & $23.3+1-0.37$ \\
\hline Radius of gyration $\boldsymbol{R}_{g}[\AA]$ & 84.2 \\
\hline Maximum extension $X[\mathrm{~nm}]$ & 29.46 \\
\hline Maximum extension $Y[n m]$ & 10.86 \\
\hline Maximum extension $\mathrm{Z}[\mathrm{nm}]$ & 9.13 \\
\hline$\%$ Helix & 9.2 \\
\hline$\%$ Sheet & 25.5 \\
\hline External links & UniProt $\theta$ AlphaFold $\theta$ \\
\hline Downloads & 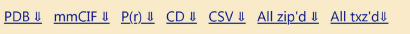 \\
\hline
\end{tabular}

13 Figure 1 - A partial screenshot of the US-SOMO-AF webpage. See Fig. 2 for the bottom half 14 containing graphs and a JSmol rendering of the structure. 
In Fig. 1, a screenshot of the US-SOMO-AF webpage (https://somo.genapp.rocks) is shown, with the bottom containing the graphic output removed (see Fig. 2). The header contains hyperlinks to the US-SOMO, SESCA, and AlphaFold websites, and to ref. ${ }^{22}$. By hovering the mouse over entries, an explanation will appear below the corresponding field on the right column. A UniProt accession number, or some initial part of it, can be entered in the first field (typing just the initial part and clicking "Search" will provide a list of corresponding entries). In any case, if the code is present in the database, the corresponding entry will be shown in the "AlphaFold model name" field, followed by the "Title" and "Source" fields as retrieved from the PDB file. If a signal peptide was identified and its coordinates removed from the PDB file, the stretch of residues involved will be listed in the "Signal peptide removed" field, otherwise "n/a" will appear. The dates on which the AF predictions and US-SOMO/SESCA computations were performed appear in their corresponding fields, and in between the "Mean confidence" field reports the calculated mean \% per-residue confidence, based on the values present in the AF-generated PDB file.

The following ten fields report the US-SOMO computed parameters. Since the hydrodynamic parameters were computed with the statistically-based ZENO method ${ }^{27,28,29}$ (see Methods), standard deviations (SD) can be generated. However, a SD is reported only for $[\eta]$, as they are tiny for all other parameters. Note that a calculated $\bar{v}$ is provided because besides being used to compute $s_{(20, w)}^{0}$ from $D_{t(20, w)}^{0}$ and $M$, it could also be used to compute an experimental $M$ from SAXS data ${ }^{16}$. The bottom two entries report the per-residue $\%$ of $\alpha$-helix and $\beta$-sheet as calculated from HELIX and SHEET fields in the cured PDB. They could be compared with CD-derived values, besides comparing experimental and calculated spectra (see below). External links for the current entry to both UniProt and AlphaFold websites are placed after the parameters listings. Cured PDB- and mmCIF-formatted files for the entry can be retrieved from the provided hyperlinks, as well as text files with the $p(r)$ vs. $r$ distribution and CD spectrum, and a csv-formatted file containing all the identifying information and the single-value parameters. All these files can be also retrieved as single compressed files (zip or tar.xz). Below these hyperlinks, the computed $p(r)$ vs. $r$ distribution and CD spectrum graphs are presented, followed by a JSmol 
1 (https://sourceforge.net/projects/jsmol) representation of the structure (see Fig. 2).

2 Controls for the visualization and copying as an image of both graphs are provided. JSmol

3 commands are also available to change the representation and export it. The default representation

4 colors the structure according to the per-residue confidence level (red, lowest; blue, highest), but for

5 a more in-depth analysis we refer the user to the original AF website.

6 In the end, parameters for a total of 365,198 structures were generated from the AF databases (that

7 include multiple predicted segments for certain sequences), and are stored in the freely accessible

8 US-SOMO-AF database.

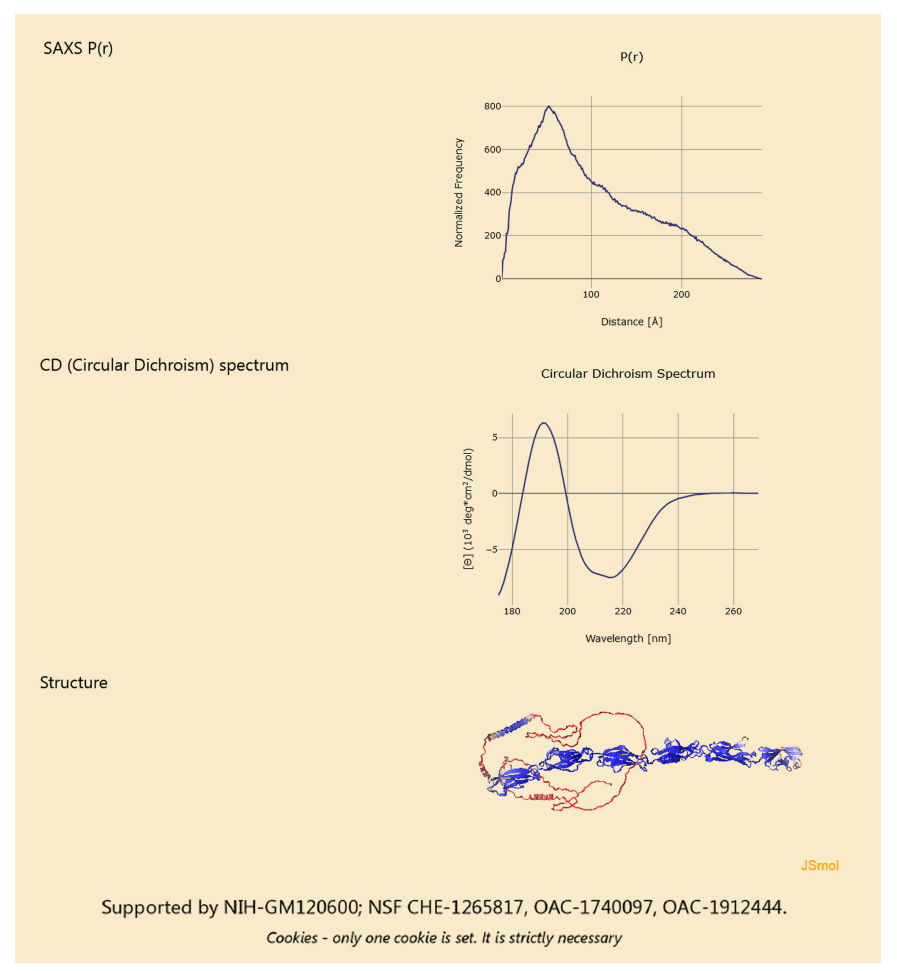

9 Figure 2 - The bottom part of the US-SOMO-AF website page showing the computed $p(r)$ vs. $r$ distribution and CD spectrum plots, and the JSmol representation of the structure.

General data analyses

13 Although it is beyond the scope of this work to provide extensive data analyses and interpretations, 14 some observations can be made. To this end, we have randomly selected from the 365,198 cured 15 structures present in the US-SOMO-AF database, a subset containing 41,200 predicted structures 16 with no counterparts in the RCSB PDB ${ }^{30}$ database (https://www.rcsb.org), and we have analyzed 17 their calculated properties (data provided as a spreadsheet, Supplementary Data 1). 

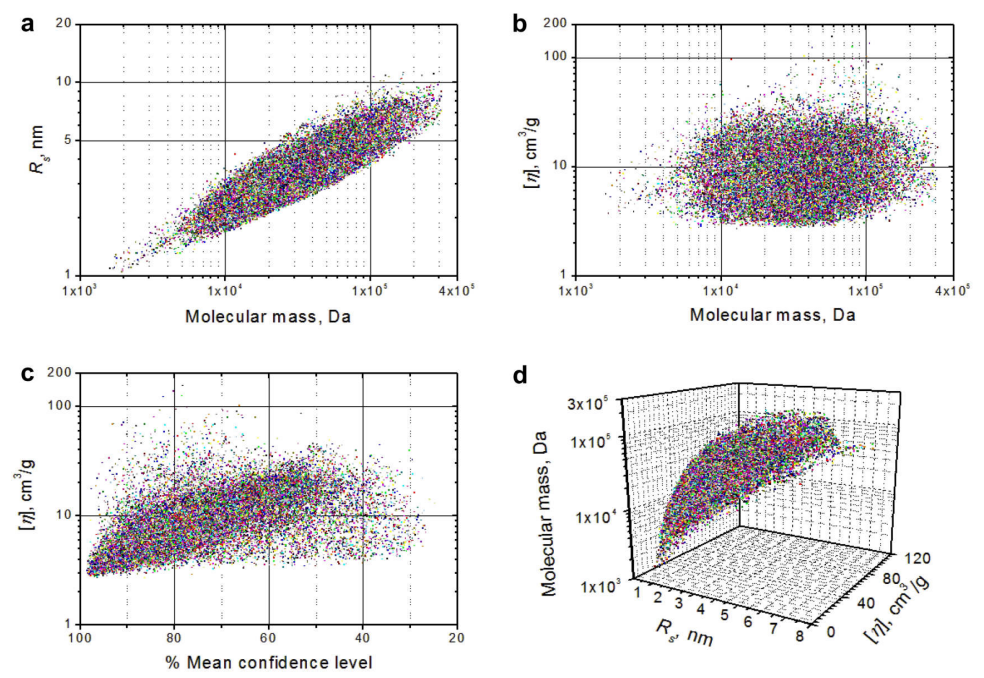

1 Figure 3 - Plots of selected calculated parameters for 41,200 predicted structures with no corresponding entries in the solved structures PDB database, randomly-extracted from the 365,198 present in the US-SOMO-AF database. Panel a, $R_{s}$ vs. $M$, log-log scale. Panel b, $[\eta]$ vs. $M, \log -\log$ scale. Panel c $[\eta]$ vs. \% decreasing mean confidence level, log-lin scale. Panel d, a 3D plot where $M$ (log scale) is on the vertical $Z$-axis, and $R_{s}$ and $[\eta]$ are on the horizontal $X$-and $Y$-axes, respectively (both linear scales).

The graphs in Fig. 3 allow for testing the discriminatory power of selected calculated parameters, by observing the spread of the $R_{s}$ (Fig. 3a) and [ $\eta$ ] (Fig. 3b) values for a given $M$ value $\left(R_{s}\right.$ was chosen as a proxy for either $s_{(20, w)}^{0}$ or $D_{t(20, w)}^{0}$, the experimentally determined parameters). It is 11 evident that $R_{s}$ alone (Fig. 3a) can already distinguish between structures, and its power, albeit somewhat limited, does not substantially change on increasing $M$ in the interval $10^{4}-10^{5} \mathrm{Da}$. A significantly larger discriminatory power is instead displayed by [ $\eta]$, almost independently of $M$ (Fig. $3 \mathbf{b}) .[\eta]$ is, however, more affected by potentially flexible regions not properly taken into account by the computations, sometimes leading to suspiciously very high values. Indeed, a correlation between increasing $[\eta]$ values vs. a decreasing \% confidence level in the structure prediction can be seen in Fig. 3c, becoming, however, much less defined when the confidence level goes below $50 \%$. Fig. $3 \mathbf{d}$ shows in 3D how combining two parameters, $R_{s}$ and [ $\eta$ ], can effectively yield an increased discriminatory power. Another important parameter is $R_{g}$, but it can rarely be determined by MALS techniques, that have a lower detection limit of $\sim 10-11 \mathrm{~nm}$. While SAXS can 
1 determine $R_{g}$, it can also be used to derive the $p(r)$ vs. $r$ distribution ${ }^{16}$, which contains more

2 information and can be directly compared with the one computed from structure. Note that the

3 effect of not taking into account the hydration water in the computation of the $p(r)$ vs. $r$ distribution

4 is relatively minor, and its importance decreases as $M$ increases. Therefore, plots involving $R_{g}$ are

5 not presented here, but could be easily generated from the Supplementary Data 1 spreadsheet.

6

$7 \quad$ Some examples

8 Table 1 - Some calculated parameters for a selection of AF-predicted structures with no RCSB PDB

9 counterparts, ordered by decreasing molecular mass. The corresponding structures and calculated

$10 p(r)$ vs. $r$ distributions and CD spectra can be seen in Fig. 4.

\begin{tabular}{l|l||c|c|c|c|c|c|c|c}
$\begin{array}{l}\text { UniProt } \\
\text { Accession }\end{array}$ & Organism & $\begin{array}{c}\text { Mean AF } \\
\text { \% conf. }\end{array}$ & $\begin{array}{c}\text { Signal } \\
\text { peptide }\end{array}$ & $\begin{array}{c}\text { Molecular } \\
\text { mass [Da] }\end{array}$ & $\begin{array}{c}R_{g} \\
{[\mathrm{~nm}]}\end{array}$ & $\begin{array}{c}R_{s} \\
{[\mathrm{~nm}]}\end{array}$ & $\begin{array}{c}{[\eta]} \\
{\left[\mathrm{cm}^{3} / \mathrm{g}\right]}\end{array}$ & $\begin{array}{c}\text { Helix } \\
\%\end{array}$ & $\begin{array}{c}\text { Sheet } \\
\%\end{array}$ \\
\hline \hline Q6PGP7 $^{\mathrm{a}}$ & H. Sapiens & 86.48 & $\mathrm{n} / \mathrm{a}$ & 175,523 & 6.98 & 6.30 & 10.4 & 74.5 & 0.5 \\
Q4DE01 $^{\mathrm{b}}$ & T. Cruzi & 65.88 & $\mathrm{n} / \mathrm{a}$ & 102,098 & 3.99 & 5.74 & 12.0 & 6.5 & 23.2 \\
Q9Y5H4 $^{\mathrm{c}}$ & H. Sapiens & 75.64 & $1-28$ & 98,141 & 8.42 & 6.56 & 23.3 & 9.2 & 25.5 \\
D3ZV97 $^{\mathrm{d}}$ & R. Norvegicus & 82.81 & $1-20$ & 94,123 & 5.55 & 4.76 & 8.93 & 42.8 & 11.2 \\
O88338 $^{\mathrm{e}}$ & M. Musculus & 84.24 & $1-21$ & 87,414 & 8.69 & 5.87 & 21.2 & 5.7 & 32.5 \\
Q9LMT9 $^{\mathrm{f}}$ & A. Thaliana & 78.02 & $1-26$ & 82,090 & 5.16 & 4.66 & 8.96 & 25.4 & 15.2 \\
I1LDW0 $^{\mathrm{g}}$ & Glycine Max & 75.28 & $\mathrm{n} / \mathrm{a}$ & 73,181 & 2.86 & 4.16 & 6.33 & 32.6 & 8.9 \\
A4I8P1 $^{\mathrm{h}}$ & L. Infantum & 60.77 & $\mathrm{n} / \mathrm{a}$ & 64,586 & 2.66 & 3.72 & 5.18 & 29.2 & 9.2 \\
Q6PFT0 $^{\mathrm{i}}$ & Danio Rerio & 81.28 & $\mathrm{n} / \mathrm{a}$ & 46,965 & 11.3 & 5.75 & 47.8 & 66.0 & 10.8 \\
Q9VG48 $^{\mathrm{j}}$ & D. Melanog. & 88.50 & $1-18$ & 44,673 & 2.04 & 2.89 & 3.44 & 38.0 & 9.0 \\
A0A060D4L2 $^{\mathrm{k}}$ & Zea Mays & 68.46 & $\mathrm{n} / \mathrm{a}$ & 30,921 & 3.90 & 4.06 & 15.7 & 32.2 & 10.4 \\
Q8IJG3 $^{1}$ & P. Falciparum & 69.83 & $\mathrm{n} / \mathrm{a}$ & 19,460 & 2.05 & 2.57 & 5.66 & 26.4 & 11.9 \\
P08372 $^{\mathrm{m}}$ & E. Coli & 82.28 & $\mathrm{n} / \mathrm{a}$ & 12,010 & 2.89 & 2.48 & 10.5 & 44.3 & 21.7 \\
O16446 $^{\mathrm{n}}$ & C. Elegans & 88.31 & $1-19$ & 8,483 & 1.18 & 1.66 & 3.46 & 68.9 & 0.0
\end{tabular}

$11{ }^{\mathrm{a}}$ Tetratricopeptide repeat protein $37 ;{ }^{\mathrm{b}}$ Trans-sialidase, putative; ${ }^{\mathrm{c}}$ Protocadherin gamma-a1;

12 ' ${ }^{\mathrm{d}}$ omeronasal 2 receptor, 50; ${ }^{\mathrm{e}}$ Cadherin-16; ${ }^{\mathrm{f}}$ Putative wall-associated receptor kinase-like 13;

13 ${ }^{\mathrm{g}}$ Aminotran_5 domain-containing protein; ${ }^{\mathrm{h}}$ Adenosine deaminase-like protein; ${ }^{\mathrm{i}}$ Flotillin; ${ }^{\mathrm{j}}$ Lipase;

$14{ }^{\mathrm{k}} \mathrm{BHLH}$ transcription factor; ${ }^{1} \mathrm{RNA}$-binding protein, putative; ${ }^{\mathrm{m}}$ Prepilin peptidase-dependent protein $15 \mathrm{C} ;{ }^{\mathrm{n}}$ Uncharacterized protein. 
1 In Table 1, we have listed 14 entries chosen from the 41,200 mentioned above. They were initially selected to represent intervals from 2.2 to 0.66 in the computed $R_{g} / R_{S}$ ratio indicating deviation from

3 globular shape $\left(R_{g} / R_{S} \sim 0.7\right.$ for a sphere). A suitable range of [ $\left.\eta\right]$ values was also sought, as well as a good representation of the organisms present in the AF databases, the presence or absence of a signal peptide, and some spread in the mean $\%$ confidence. $M, R_{g}, R_{s}$, and [ $\eta$ ] were chosen as the calculated parameters, and the entries are ordered by decreasing $M$. Connected to Table 1 is Fig. 4 , that displays snapshots of the 3D structures for each entry colored according to the per-residue confidence level, followed by the $p(r)$ vs. $r$ and CD plots.

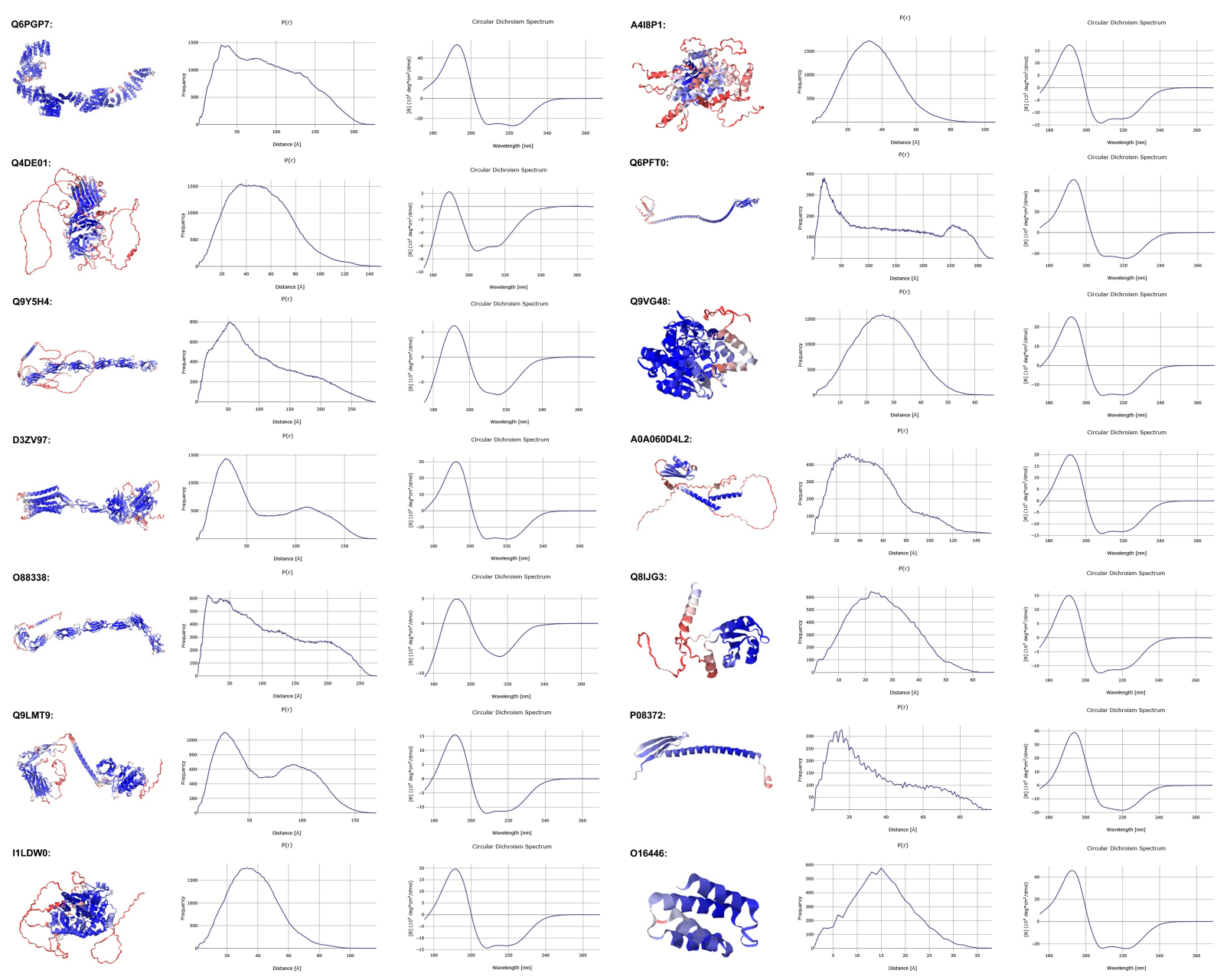

9 Figure 4 - JSmol snapshots of the structures for the entries reported in Table 1, together with the calculated $p(r)$ vs. $r$ and CD plots.

Table 1 and Fig. 4 provide an insightful glimpse on the great variety of predicted structures and their associated calculated parameters, suggesting that performing some of these checks can indeed boost, or question, their reliability. As expected, CD spectra display differences between most 
structures, and they are a robust check on the predicted secondary structure content. The variability in $[\eta]$ values in Table 1 appears to confirm its discriminating power above that of $R_{s}$, but clearly it is the $p(r)$ vs. $r$ distribution that would provide the best test, although it is the least rapidly experimentally accessible parameter among those considered.

To provide an additional measure of the predictive power of the hydrodynamic parameters and of the $p(r)$ vs. $r$ distribution, we have selected the 088338 Cadherin-16 from M. Musculus structure (see Table 1 and Fig. 4) that contains a number of independently folded domains connected by linkers, and we have run a Discrete Molecular Dynamics (DMD) ${ }^{31,32}$ simulation to expand its conformational space (see Online Methods for details), followed by hydrodynamic and $p(r)$ vs. $r$ calculations on 100 produced structures. As can be seen in the Supplementary Video 1, sufficiently different alternative conformations were generated within an overall frame, allowing an evaluation of the spread in the predicted parameters and their potential discriminating power. For instance, the $R_{s}$ spread, 5.88-6.16 nm (a $\sim 4.5 \%$ change) would be barely above experimental error in distinguishing between the most different conformations in this set, while the spread in $[\eta]$, $20.8-23.8 \mathrm{~cm}^{3} / \mathrm{g}$ ( $\sim 12.6 \%$ change), would clearly allow distinguishing between many conformations (all this set's individual data are in Supplementary Data 2 spreadsheet, and the $R_{s}$ and $[\eta]$ are reported in each video frame, along with the $p(r)$ vs. $r$ distributions). Even more striking is the variation in the $p(r)$ vs. $r$ distributions that are also collectively reported in Fig. 5.

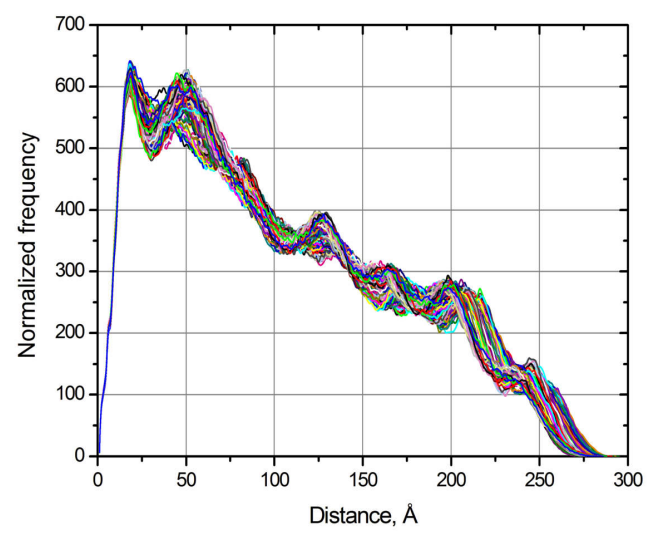

19 Figure 5 - Calculated $p(r)$ vs. $r$ distributions for all the conformations generated in the DMD run on the AF-predicted O88338 structure.

21 Thus, even for such a restricted structural variation, comparing experimental and calculated parameters can provide reliable tests of the predicted structures. 


\section{Discussion}

2 We have presented here a new database stemming from the AlphaFold predicted protein structures database. It contains calculated hydrodynamic and structural parameters whose experimental determination should be within the reach of scientists working with a particular protein for which a "hard" structure is either currently unavailable or in the making. Indeed, it is interesting to note that crystallographers and cryo-electron microscopists are already suggesting using AF-predicted structures to solve experimental structures by molecular replacement methods ${ }^{33}$. Performing some rapid tests and comparing the results with those we provide in the US-SOMO-AF database could save them valuable time and perhaps hint at twists that should be applied to a predicted structure to better fit the X-ray, cryo-EM, and NMR data. In this respect, we would like to point out a tool

11 present in the US-SOMO program that allows one to color-code a visualized structure based on the contribution of residues to a particular set of distances in a $p(r)$ vs. $r$ distribution ${ }^{34}$. For instance, this could provide an easier identification of domains that under- or over-contribute to that set of distances. This is another reason why we chose to produce $p(r)$ vs. $r$ distributions instead of simulated SAXS intensity vs. scattering vector curves, for which a wide variety of methods, often quite computationally intensive, exist ${ }^{35}$. More in-depth analyses could be subsequently performed on case-by-case basis.

For a more general use, assessing the reliability of a predicted structure could lead to better designed function/structure relationship experiments. The availability of the US-SOMO-AF database has the distinctive advantage of allowing a quick comparison without the need to master the expertise necessary to soundly calculate the relevant solution parameters. There are, of course, a series of drawbacks associated with these computations. First and foremost, all the AF predicted structures consider all proteins as single chain entities. Efforts are apparently underway $\left(\mathrm{see}^{5}\right)$ to cope with this issue by allowing multi-chain predictions, and when an evolution in that sense appears in the AF database, we can re-calculate all parameters for a new set. A second evident drawback resides in the post-translational modifications that many proteins undergo. None were considered by the AF team, and we have just scratched the surface by removing the signal 
1 peptides. The most important modification, affecting the calculated parameters, is glycosylation

2 (e.g., see Table $1 \mathrm{in}^{36}$ ). While UniProt provides a list of potential glycosylation sites for entries, and

3 publications describing them when available, there is no direct way to have the composition of each

4 carbohydrate associated with a particular site. This is a pity, as methods for building complex

5 carbohydrates are already available and/or under development $\left(\mathrm{see}^{37}\right)$, and it should be relatively

6 straightforward to automatically add them at the appropriate sites. Indeed, this has just been

7 independently advocated in a very recent letter to this journal ${ }^{38}$. Even in absence of time-consuming

8 molecular dynamics minimization steps, this simple addition could increase the reliability of

9 calculated hydrodynamic and structural parameters. While we hope that such an important step will

10 be taken at the UniProt database level, users that need to refine the calculations on a predicted

11 structure after having manually added any prosthetic group can easily do so by using one of the

12 downloadable (http://somo.aucsolutions.com) US-SOMO versions.

13 The third drawback is the handling of flexibility, especially if large unstructured parts are predicted.

14 Here the US-SOMO-AF database can only raise red flags, such as very high predicted $[\eta]$ values

15 associated with visualized extended, unstructured parts. Dealing with these issues requires much

16 longer calculations involving either Monte Carlo methods or Brownian dynamics simulations

$17\left(\mathrm{see}^{39}\right)$, that would require a major effort to be applied systematically on $>365,000$ structures.

18 All considered, we believe that the publicly available (https://somo.genapp.rocks) US-SOMO-AF

19 database described here will become a useful tool allowing the research community, by comparing

20 one or more experimentally-determined parameters with the corresponding computed ones, to

21 quickly evaluate the compatibility in solution of an AlphaFold-predicted protein structure.

Methods

24 Production of the results presented in this paper required five major steps: collect the AlphaFold entries and additional metadata; prepare the structures for hydrodynamic, structural and CD calculations; compute the hydrodynamic, structural and CD properties; build a database containing the hydrodynamic properties and additional metadata; and finally build a website allowing users 
convenient access to the database.

After downloading the AlphaFold database, we prepared the structures by removing the signal peptide regions, where present, identified from the UniProt website. We utilized US-SOMO ${ }^{21,22,23}$ to compute hydrodynamic and structural properties. The US-SOMO suite uses a bead modeling strategy which takes into account the theoretical amount of bound hydration water, and the ZENO computational algorithm ${ }^{27,28,29}$ was employed to calculate the hydrodynamic parameters in a rigidbody frame. US-SOMO was also used to compute the $\mathrm{p}(\mathrm{r})$ vs. $\mathrm{r}$ distribution on not-hydrated structures, using SAXS-related parameters. To compute the CD spectra, we used SESCA ${ }^{18}$. All the computed results were collected and inserted into a database.

Full descriptions for all these steps can be found in the Supplementary Methods section.

\section{Acknowledgements}

Research reported in this publication was supported by the NIGMS of the National Institutes of Health under award number R01GM120600 and by the National Science Foundation under awards CHE-1265817, OAC-1740097, OAC-1912444, all to E.B. The content is solely the responsibility of the authors and does not necessarily represent the official views of the National Institutes of Health nor the National Science Foundation. This work used the Extreme Science and Engineering Discovery Environment (XSEDE), which is supported by National Science Foundation grant number ACI-1548562 and utilized Jetstream at Indiana University and the Texas Advanced Computer Center through allocation TG-MCB17057 to EB. We are grateful to P. Vachette (I2BC, Université Paris-Saclay, CEA, CNRS, Gif-sur-Yvette, France) for comments and suggestions.

\section{Authors contributions}

MR conceived the outline of the work, analyzed results, and wrote the paper. EB contributed to define the work, developed all software, performed all computational and website implementation tasks, and wrote the paper. 


\section{Competing Financial Interests}

2 The Authors declare no competing financial interests.

\section{Data Availability Statement}

The datasets generated during and/or analyzed during the current study are available in the US-SOMO-AF website, https://somo.genapp.rocks.

\section{References}

1. Anfinsen, C.B. Principles that govern the folding of protein chains. Science 181, 223-230 (1973) Doi: https://doi.org/10.1126/science.181.4096.223

2. Jumper, J. et al. Highly accurate protein structure prediction with AlphaFold. Nature 596, 583589 (2021). Doi: https://doi.org/10.1038/s41586-021-03819-2

3. Baek, M. et al. Accurate prediction of protein structures and interactions using a three-track neural network. Science 373, 871-876 (2021). Doi: https://doi.org/10.1126/science.abj8754

4. Tunyasuvunakool, K. et al. Highly accurate protein structure prediction for the human proteome. Nature 596, 590-596 (2021). Doi: https://doi.org/10.1038/s41586-021-03828-1

5. Perrakis, A. \& Sixma, T.K. AI revolutions in biology: The joys and perils of AlphaFold. EMBO Rep. e54046 (2021). Doi: https://doi.org/10.15252/embr.202154046

6. Pelton, J.T. \& McLean, L.R. Spectroscopic methods for analysis of protein secondary structure. Anal. Biochem. 277, 167-176 (2000). Doi: https://doi.org/10.1006/abio.1999.4320

7. Unzai, S. Analytical ultracentrifugation in structural biology. Biophys. Rev. 10, 229-233 (2018). Doi: https://doi.org/10.1007/s12551-017-0340-0

8. Methods Enzymol. Analytical Ultracentrifugation. Edited by James L. Cole. Volume 562, pages 2-567 (2015).

9. Some, D., Amartely, H., Tsadok, A. \& Lebendiker, M. Characterization of proteins by SizeExclusion Chromatography coupled to Multi-Angle Light Scattering (SEC-MALS). J. Vis. Exp. 148, e59615 (2019). Doi: https://doi.org/10.3791/59615 
10. Stetefeld, J., McKenna, S.A. \& Patel, T.R. Dynamic light scattering: a practical guide and applications in biomedical sciences. Biophys Rev. 8, 409-427 (2016).

Doi: https://doi.org/10.1007/s12551-016-0218-6

11. https://wyattfiles.s3-us-west-2.amazonaws.com/literature/app-notes/dls-plate/WP5003-Automated-dynamic-and-static-light-scattering-in-microwell-plates.pdf

12. Harding, S.E. The intrinsic viscosity of biological macromolecules. Progress in measurement, interpretation and application to structure in dilute solution. Prog. Biophys. Mol. Biol. 68, 207262 (1997). Doi: https://doi.org/10.1016/s0079-6107(97)00027-8

13. Striegel, A.M. Viscometric detection in size-exclusion chromatography: Principles and select applications. Chromatographia 79, 945-960 (2016).

Doi: https://doi.org/10.1007/s10337-016-3078-0

14. Glatter, O. A new method for the evaluation of small-angle scattering data. J. Appl. Cryst. 10, 415-421 (1977) Doi: https://doi.org/10.1107/S0021889877013879

15. Bizien, T. et al. A brief survey of state-of-the-art BioSAXS. Protein Pept. Lett. 23, 217-231 (2016). Doi: $\underline{\text { https://doi.org/10.2174/0929866523666160106153655 }}$

16. Jeffries, C. et al. Small-angle X-ray and neutron scattering. Nat. Rev. Methods Primers 1, 70 (2021). Doi: https://doi.org/10.1038/s43586-021-00064-9

17. Pérez, J. \& Vachette, P. A successful combination: Coupling SE-HPLC with SAXS. In: Biological Small Angle Scattering: Techniques, Strategies and Tips. Chaudhuri, B., Muñoz, I., Qian, S. \& Urban, V. (eds). Advances in Experimental Medicine and Biology, 1009. Springer, Singapore (2017). Doi: https://doi.org/10.1007/978-981-10-6038-0_11

18. Nagy, G., Igaev, M., Jones, N.C., Hoffmann, S.V. \& Grubmüller, H. SESCA: Predicting circular dichroism spectra from protein molecular structures. J. Chem. Theory Comput. 15, 5087-5102 (2019). Doi: https://doi.org/10.1021/acs.jctc.9b00203

19. Rocco, M. \& Byron, O. Computing translational diffusion and sedimentation coefficients: an evaluation of experimental data and programs. Eur. Biophys. J. 44, 417-431 (2015). Erratum in: Eur. Biophys. J. 44, 433-436 (2015). Doi: https://doi.org/10.1007/s00249-015-1042-9 
20. Rocco, M. \& Byron, O. Hydrodynamic modeling and its application in AUC. Methods Enzymol. 562, 81-108 (2015). Doi: https://doi.org/10.1016/bs.mie.2015.04.010

21. Brookes, E., Demeler, B., Rosano, C. \& Rocco, M. The implementation of SOMO (SOlution MOdeller) in the UltraScan analytical ultracentrifugation data analysis suite: enhanced capabilities allow the reliable hydrodynamic modeling of virtually any kind of biomacromolecule. Eur. Biophys J. 39, 423-435 (2010). Doi: https://doi.org/10.1007/s00249-009-0418-0

22. Brookes, E. \& Rocco, M. Recent advances in the UltraScan SOlution MOdeller (US-SOMO) hydrodynamic and small-angle scattering data analysis and simulation suite. Eur. Biophys. J. 47, 855-864 (2018). Doi: https://doi.org/10.1007/s00249-018-1296-0

23. Rocco, M., Brookes, E. \& Byron, O. US-SOMO: Methods for construction and hydration of macromolecular hydrodynamic models. In: Encyclopedia of Biophysics, Roberts, G. \& Watts, A., European Biophysical Societies (eds). Springer, Berlin, Heidelberg (2021). Doi: https://doi.org/10.1007/978-3-642-35943-9_292-1

24. Owji, H., Nezafat, N., Negahdaripour, M., Hajiebrahimi, A. \& Ghasemi, Y. A comprehensive review of signal peptides: Structure, roles, and applications. Eur. J. Cell Biol. 97, 422-441 (2018). Doi: https://doi.org/10.1016/j.ejcb.2018.06.003

25. Kabsch, W. \& Sander, C. Dictionary of protein secondary structure: pattern recognition of hydrogen-bonded and geometrical features. Biopolymers 22, 2577-2637 (1983). Doi: https://doi.org/10.1002/bip.360221211

26. Pettersen, E.F. et al. UCSF Chimera--a visualization system for exploratory research and analysis. J. Comput. Chem. 25, 1605-1612 (2004). Doi: https://doi.org/10.1002/jcc.20084 27. Kang, E.H., Mansfield, M.L. \& Douglas, J.F. Numerical path integration technique for the calculation of transport properties of proteins. Phys. Rev. E Stat. Nonlinear Soft Matter Phys. 69, 031918 (2004). Doi: https://doi.org/10.1103/PhysRevE.69.031918 
28. Mansfield, M.L. \& Douglas, J.F. Improved path integration method for estimating the intrinsic viscosity of arbitrarily shaped particles. Phys. Rev. E Stat. Nonlinear Soft Matter Phys. 78, 046712 (2008). Doi: https://doi.org/10.1103/physreve.78.046712

29. Juba, D., Audus, D.J., Mascagni, M., Douglas, J.F. \& Keyrouz, W. ZENO: Software for calculating hydrodynamic, electrical, and shape properties of polymer and particle suspensions. J. Res. Natl. Inst. Stand. Technol. 122, 1-2 (2017). Doi: https://doi.org/10.6028/jres.122.020

30. Berman, H.M. et al. The Protein Data Bank. Nucleic Acids Res. 28, 235-242 (2000). Doi: https://doi.org/10.1093/nar/28.1.235

31. Dokholyan, N.V., Buldyrev, S.V., Stanley, H.E. \& Shaknovich, E.I. Discrete molecular dynamics studies of the folding of a protein-like model. Folding \& Design 3, 577-587 (1998). Doi: https://doi.org/10.1016/s1359-0278(98)00072-8

32. Ding, F. \& Dokholyan, N,V. Emergence of protein fold families through rational design. PLoS Comput. Biol. 2, e85 (2006). Doi: https://doi.org/10.1371/journal.pcbi.0020085

33. Kryshtafovych, A. et al. Computational models in the service of X-ray and cryo-electron microscopy structure determination. Proteins (2021) Epub ahead of print. Doi: https://doi.org/10.1002/prot.26223

34. Brookes, E. et al. Fibrinogen species as resolved by HPLC-SAXS data processing within the UltraScan Solution Modeler (US-SOMO) enhanced SAS module. J. Appl. Crystallogr. 46, 1823-1833 (2013). Doi: https://doi.org/10.1107/S0021889813027751

35. Hub, J.S. Interpreting solution X-ray scattering data using molecular simulations. Curr. Opin. Struct. Biol. 49, 18-26 (2018). Doi: https://doi.org/10.1016/j.sbi.2017.11.002

36. Rocco, M., Rosano, C., Weisel, J.W., Horita, D.A. \& Hantgan, R.R. Integrin conformational regulation: uncoupling extension/tail separation from changes in the head region by a multiresolution approach. Structure 16, 954-964 (2008). Doi: https://doi.org/10.1016/j.str.2008.02.019 
1 37. Agirre, J. Strategies for carbohydrate model building, refinement and validation. Acta Crystallogr. D Struct. Biol. 73, 171-186 (2017). Doi: $\underline{\text { https://doi.org/10.1107/S2059798316016910 }}$

38. Bagdonas, H., Fogarty, C.A., Fadda, E. \& Agirre, J. The case for post-predictional modifications in the AlphaFold Protein Structure Database. Nat. Struct. Mol. Biol. 28, 869-870 (2021). Doi: https://doi.org/10.1038/s41594-021-00680-9

39. García de la Torre, J., Ortega, A., Amorós, D., Rodríguez Schmidt, R. \& Hernández Cifre, J.G.

8 Methods and tools for the prediction of hydrodynamic coefficients and other solution properties

9 of flexible macromolecules in solution. A tutorial minireview. Macromol. Biosci. 10, 721-730 (2010). Doi: https://doi.org/10.1002/mabi.200900464 


\section{Figures Legends}

2 Figure 1 - A partial screenshot of the US-SOMO-AF webpage. See Fig. 2 for the bottom half 3 containing graphs and a JSmol rendering of the structure.

4 Figure 2 - The bottom part of the US-SOMO-AF website page showing the computed $p(r)$ vs. $r$

5 distribution and CD spectrum graphs, and the JSmol representation of the structure.

6 Figure 3 - Plots of selected calculated parameters for 41,200 predicted structures with no corresponding entries in the solved structures PDB database, randomly-extracted from the 365,198

8 present in the US-SOMO-AF database. Panel a, $R_{s}$ vs. $M, \log -\log$ scale. Panel $\mathbf{b},[\eta]$ vs. $M, \log -\log$

9 scale. Panel c $[\eta]$ vs. \% decreasing mean confidence level, log-lin scale. Panel d, a 3D plot where $M$

10 (log scale) is on the vertical $Z$-axis, and $R_{s}$ and $[\eta]$ are on the horizontal $X$ - and $Y$-axes, respectively 11 (both linear scales).

12 Figure 4 - JSmol snapshots of the structures for the entries reported in Table 1, together with the 13 calculated $p(r)$ vs. $r$ and CD plots.

14 Figure 5 - Calculated $p(r)$ vs. $r$ distributions for all the conformations generated in the DMD run on 15 the AF-predicted O88338 structure. 


\section{Supplementary Files}

This is a list of supplementary files associated with this preprint. Click to download.

- NSRBrookesRoccoSuppData1.xlsx

- NSRBrookesRoccoSuppData2.xlsx

- NSRBrookesRoccoSupplementaryVideo1.mp4

- NSRBrookesRoccoSupplementarylnfo.pdf 